\title{
Visibility Number of Directed Graphs
}

\author{
Maria Axenovich*, Andrew Beveridge ${ }^{\dagger}$, Joan P. Hutchinson ${ }^{\dagger}$, Douglas B. West ${ }^{\S}$
}

\begin{abstract}
A $k$-bar visibility representation of a digraph $G$ assigns each vertex at most $k$ horizontal segments in the plane so that $G$ has an arc $u v$ if and only if some segment for $u$ "sees" some segment for $v$ above it by a vertical line of sight. The (bar) visibility number $b(G)$ of a digraph $G$ is the least $k$ permitting such a representation. Among other results, we show that $b(G) \leq 4$ when $G$ is a planar digraph (reducing to 3 when the underlying graph has no triangles), $b(G) \leq 2$ when $G$ is outerplanar, and $b(G) \leq(n+10) / 3$ when $G$ has $n$ vertices. When $G$ is the $n$-vertex transitive tournament, $b(G) \leq 7 n / 24+2 \sqrt{n \log n}$, improving to $b(G)<3 n / 14+42$ when $n$ is sufficiently large. Our tools include arboricity, interval number, and Steiner systems.
\end{abstract}

\section{Introduction}

Among representations of undirected graphs, researchers have studied many geometric models in which edges correspond to clear lines of sight joining regions that represent vertices. For example, a bar-visibility representation assigns each vertex a horizontal segment (a bar) in the plane, and two vertices are adjacent if and only their bars can "see" each other. A line of sight is a channel of positive width joining two objects in the plane that is disjoint from other objects corresponding to vertices. A graph having a bar-visibility representation is a bar-visibility graph. Bar-visibility graphs must be planar; they have been characterized, applied, and studied from both theoretical and algorithmic viewpoints.

Such line-of-sight graphs may have been studied first in [8], where a graph related to a printed circuit board was checked for "short circuits" using a graph-coloring result. When

*Department of Mathematics, Iowa State University, Ames, IA 50011: axenovic@iastate.edu. Present address: Department of Mathematics, Karlsruhe Institute of Technology, D-76128 Karlsruhe, Germany. Research partially supported by NSF grant DMS-0901008

${ }^{\dagger}$ Department of Mathematics, Statistics and Computer Science, Macalester College, Saint Paul, MN 55105: abeverid@macalester.edu

${ }_{\ddagger}^{\ddagger}$ Department of Mathematics, Statistics and Computer Science, Macalester College, Saint Paul, MN 55105: hutchinson@macalester.edu

$\S$ Department of Mathematics, University of Illinois, Urbana, IL 61801: west@math.uiuc.edu. Research supported by NSA grant H98230-10-1-0363. 
the assigned sets are rectangles and both horizontal and vertical visibilities are allowed, we obtain rectangle-visibility graphs, relevant to VLSI chip layout and "two-layer routing" $[29,14,5]$. In general, visibility graphs are related to graph drawing and find applications in computational geometry. Early papers on bar-visibility graphs include [31, 27, 32, 17]; surveys and overviews can be found in [2, 9, 23].

Since bar-visibility representations exist only for some planar graphs, it is natural to introduce more flexibility to permit representations of all graphs. A $k$-bar visibility representation of a graph assigns each vertex at most $k$ bars in the plane so that vertices $u$ and $v$ are adjacent if and only if some bar for $u$ (called a $u$-bar) and some bar for $v$ (a $v$-bar) see each other by a vertical line of sight. The (bar) visibility number, introduced in [7], is the least $k$ permitting such a representation; it is a measure of distance from being a bar-visibility graph. In [7], extremal problems for this parameter were studied on various classes of graphs.

The corresponding concepts for directed graphs (digraphs) are also natural. A $k$-bar visibility representation or $k$-fold layout of a digraph assigns each vertex at most $k$ bars in the plane so that there is an arc $u v$ in the digraph if and only if some $u$-bar sees some $v$-bar above it by a vertical line of sight. The (bar) visibility number $b(G)$ of a digraph $G$ is the least $k$ permitting such a representation. The digraphs $G$ such that $b(G)=1$ are bar-visibility digraphs. For $k=1$ and $k=2$, we obtain single-bar and double-bar layouts, respectively.

Bar-visibility layouts of digraphs can depict hierarchical structures in flow diagrams, networks, and family trees; see [3, 2]. In this paper, we study extremal problems for visibility number in the families of planar digraphs, outerplanar digraphs, bipartite digraphs, tournaments, and general $n$-vertex digraphs.

A $k$-fold layout of a digraph is also a $k$-bar visibility representation of its underlying graph. This elementary observation relates the visibility numbers of a graph and its orientations.

Observation 1.1. If $G$ is an orientation of a graph with visibility number $k$, then $b(G) \geq k$.

We cannot guarantee that the visibility number of a graph $H$ is the minimum of the visibility numbers of its orientations, since an optimal visibility representation of $H$ may require a vertex to see another vertex both above and below. All digraphs in this paper are oriented graphs, meaning orientations of simple graphs. There are no loops or multi-edges, and at most one of $\{x y, y x\}$ is an arc. Since our focus is on digraphs, we use $G$ to denote the digraph whose visibility number we are studying, and we use $\hat{G}$ to denote the underlying undirected graph of $G$; that is, $G$ is an orientation of $\hat{G}$. When we begin with an undirected graph, we call it $H$ and may consider an arbitrary orientation $G$ of $H$.

We use "oriented tree", "oriented cycle", "oriented caterpillar", etc., for an orientation of a graph in the named family. When the family name contains the word "graph", we instead use "digraph", writing "planar digraph" (for example) for an oriented planar graph. 
From the beginning, bar-visibility digraphs were studied along with bar-visibility graphs $[2,27,31,32]$; thus the digraphs with visibility number 1 have long been known. In a digraph, a vertex is a source or a sink when it has indegree 0 or outdegree 0 , respectively. In this paper, we use the term consistent cycle to mean an oriented cycle having no source or sink.

Theorem 1.2 ([27, 32]). Given a planar digraph $G$, let $G^{\prime}$ be the digraph formed by adding two vertices $s$ and $t$, an arc sv for every source vertex $v$ in $G$, an arc wt for every sink vertex $w$, and the arc st. A planar digraph $G$ is a bar-visibility digraph if and only if $G^{\prime}$ is planar and has no consistent cycle.

For example, consider oriented cycles. Let a semi-cycle be an oriented cycle having exactly one source and one sink. An oriented cycle is a bar-visibility digraph if and only if it is a semi-cycle. If there are at least two sources and two sinks, then the underlying graph of the digraph $G^{\prime}$ tested in the criterion of Theorem 1.2 contains a subdivision of $K_{3,3}$.

In the remainder of this section, we summarize our results, postponing the proofs to later sections. By analogy with consistent cycles, let a consistent path be an oriented path that has exactly one source and one sink; a path that is not consistent is inconsistent. All oriented paths are bar-visibility digraphs, and more generally it is easy to determine the visibility numbers of oriented trees.

Proposition 1.3. If $G$ is an oriented tree, then $b(G) \leq 2$, with equality if and only if $G$ has a vertex that is an endpoint of three edge-disjoint inconsistent paths.

Once multiple bars are needed, we seek analogues of results on undirected graphs. For example, [7] showed that every planar graph has visibility number at most 2, which is sharp.

Theorem 1.4. If $G$ is a planar digraph, then $b(G) \leq 4$. If also $\hat{G}$ is triangle-free or contains no subdivision of $K_{2,3}$, then $b(G) \leq 3$. If $\hat{G}$ has girth at least 6 , then $b(G) \leq 2$.

These bounds are proved using various decompositions of the underlying graphs into forests, where a decomposition of a [di]graph $G$ is a set of sub[di]graphs such that each edge of $G$ lies in exactly one of them. We do not know whether there is a planar digraph with visibility number 4 , but we present planar digraphs (having triangles) with visibility number 3. Further increasing the girth of the underlying planar graph cannot force the visibility number below 2 , since the value is 2 for consistent cycles.

A graph is outerplanar if it has an embedding with every vertex on the unbounded face.

Theorem 1.5. If $G$ is an outerplanar digraph, then $b(G) \leq 2$. Equality holds when $G$ is a consistent cycle (and for many other digraphs). 
Decomposing a digraph $G$ into subdigraphs with small visibility number (such as oriented trees, planar digraphs, or digraphs in families with visibility number 1) can yield a good upper bound on $b(G)$ via the next observation. A component of a layout is a maximal set of bars $C$ such that every vertical line between the leftmost line intersecting $\bigcup_{b \in C} b$ and the rightmost line intersecting $\bigcup_{b \in C} b$ also intersects $\bigcup_{b \in C} b$.

Observation 1.6. If a digraph $G$ decomposes into digraphs $G_{1}, \ldots, G_{t}$, then $b(G) \leq \sum_{i} b\left(G_{i}\right)$. Proof. Lay out $G_{1}, \ldots, G_{t}$ as components of a layout of $G$.

For a digraph $G$, Observation 1.1 gives a general lower bound on $b(G)$ in terms of $\hat{G}$. Any lower bound on the visibility number of $\hat{G}$ is a lower bound for $b(G)$. For example, in [7], simple counting arguments using Euler's Formula yield lower bounds on visibility number in terms of the number of vertices and edges.

Theorem 1.7. If $G$ is a digraph with $n$ vertices and $m$ arcs, then $b(G) \geq(m+6) /(3 n)$. If also $\hat{G}$ has no triangles, then $b(G) \geq(m+4) /(2 n)$.

On the other hand, many upper bounds for undirected graphs that are proved using representations where no vertical line intersects more than two bars yield corresponding upper bounds on the visibility number of orientations of those graphs. We explore this approach in detail in Section 2, using various results on interval intersection representations of graphs. Here we simply state one such result to motivate related results.

Theorem 1.8. If $G$ is a digraph such that $\Delta(\hat{G}) \leq r$, then $b(G) \leq\left\lceil\frac{r+1}{2}\right\rceil$.

Although Theorem 1.8 is sharp for $r \leq 3$, in general we do not know the best bound on $b(G)$ in terms of the maximum degree $\Delta(\hat{G})$ of $\hat{G}$. For orientations of triangle-free regular graphs, the upper bound in Theorem 1.8 differs from the lower bound $(m+4) / 2 n$ in Theorem 1.7 by approximately a factor of 2 . We ask whether the upper bound in terms of degree is sharp for regular bipartite graphs.

Theorem 1.8 yields $b(G) \leq\lceil n / 2\rceil$ when $G$ is an $n$-vertex oriented graph. Except for very small $n$, we can improve this bound using Steiner systems of the form $S(2,4, m)$. A Steiner system $S(t, k, m)$ is a family of $k$-element subsets of an $m$-set such that any $t$ elements of the $m$-set appear together in exactly one member of the family. We do not know whether the resulting bound is sharp; again examples are known within a factor of 2 .

Theorem 1.9. If $G$ is an $n$-vertex digraph, then $b(G) \leq(n+10) / 3$.

Chang et al. [7] showed also that the visibility number of the undirected complete graph with $n$ vertices is precisely $\lceil n / 6\rceil$. The lower bound follows from Euler's Formula, while the 
upper bound uses the solution to Heawood's Empire Problem [12, 15]. By Observation 1.1, $b(G) \geq\lceil n / 6\rceil$ when $G$ is an $n$-vertex tournament, where a tournament is an orientation of a complete graph. Even when restricted to tournaments, the extremal problem remains unsolved. We do not know the maximum visibility number of $n$-vertex tournaments, but for transitive tournaments (in which having the arcs $x y$ and $y z$ requires having the arc $x z$ ), we improve the upper bound.

Theorem 1.10. If $G$ is a transitive $n$-tournament, then

(a) $b(G) \leq 7 n / 24+2 \sqrt{n \log n}$, and

(b) when $n$ is sufficiently large, $b(G)<3 n / 14+42$.

Statement (a) improves upon the construction of Theorem 1.9 using a probabilistic argument. Statement (b) uses the Steiner system $S(2,15, m)$, which exists when $m$ is sufficiently large and satisfies divisibility conditions that are obviously necessary.

In many cases, upper bounds on visibility number for orientations in certain families are twice the bound in [7] for the corresponding family of graphs. Although we believe that $b(G)$ is at most twice the visibility number of $\hat{G}$ when $G$ is an oriented graph, we have no general proof of that, and our upper bound arguments (after Section 2) require careful analysis.

\section{Visibility, Arboricity, and Interval Number}

Many of the proofs and results of this section are motivated by analogous results for interval representations of graphs. A depth-2 $k$-fold layout of a digraph $G$ is one in which every vertical line meets at most two bars of the layout. A $t$-interval is the union of at most $t$ intervals on the real line. The interval number of a graph $H$, written $i(H)$, is the least $t$ such that $H$ has a t-interval representation, which is an expression of $H$ as the intersection graph of a family of $t$-intervals. A $t$-interval representation of $H$ has depth 2 if every point of the real line lies in the sets for at most two vertices (see $[10,26]$ ). Let $i_{2}(H)$ denote the least $t$ such that $H$ has a depth- 2 -interval representation.

Proposition 2.1. A digraph $G$ has a depth-2 $k$-fold visibility layout if and only if $\hat{G}$ has a depth-2 k-interval representation.

Proof. Projecting such a layout of $G$ onto a single horizontal line yields a depth- $2 k$-interval representation of $\hat{G}$. Conversely, given such a representation, process it from left to right, converting each interval to a bar in a visibility layout. Each time a new interval is encountered, there is (at most) one other interval containing its left endpoint and only one edge 
created. Shift the new interval vertically in the plane, placing it higher or lower than the bar that arose from its earlier neighbor depending on the direction of the corresponding arc in $G$. By construction, the result is a depth- $2 k$-fold visibility layout.

With Proposition 2.1, many upper bounds on interval number of graphs translate immediately into upper bounds on visibility number of oriented graphs. Direct arguments can be given to construct the corresponding visibility layouts, but essentially they would just repeat the arguments for interval number. Nevertheless, to facilitate understanding, we briefly mention the ideas that yield the corresponding bounds on the depth- 2 interval number.

(Theorem 1.8). If $G$ is an oriented graph, then $b(G) \leq\left\lceil\frac{r+1}{2}\right\rceil$, where $r=\Delta(\hat{G})$.

Proof. For undirected graphs, Griggs and West [10] showed that $i_{2}(H) \leq\lceil(\Delta(H)+1) / 2\rceil$. The construction arises by following an Eulerian circuit in each component of the supergraph obtained by adding one vertex adjacent to all the vertices having odd degree in $H$.

The arboricity of a graph $H$, written $\Upsilon(H)$, is the minimum number of forests needed to decompose $H$. Trotter (see [26]) observed that $i(H) \leq 1+\Upsilon(H)$; this can be improved a bit. A linear forest is a forest with maximum degree at most 2 (the components are paths). The linear arboricity of a graph is the minimum size of a decomposition of $H$ into linear forests. Define the semi-linear arboricity of a graph $H$, written $\Upsilon^{\prime}(H)$, to be the minimum number of forests in a decomposition of $H$ such that at least one of the forests is linear.

Note that $\Upsilon(H) \leq \Upsilon^{\prime}(H) \leq 1+\Upsilon(H)$; the lower bound is by definition, and the upper bound holds because a linear forest with no edges can be added to an optimal decomposition. The use of semi-linear arboricity allow us sometimes to improve Trotter's bound by 1, which is significant for families whose graphs have small arboricity.

Proposition 2.2. If $H$ is an undirected graph, then $i_{2}(H) \leq \Upsilon^{\prime}(H)$. If $G$ is an orientation of $H$, then $b(G) \leq \Upsilon^{\prime}(H)$.

Proof. The second statement follows from the first by Proposition 2.1. For the first, start with the linear forest $F$ used in the decomposition. Represent each component of $F$ by intervals on the real line such that the interval for a vertex intersects only the intervals for the vertices neighboring it on the path, and for each interval there is a "displayed" portion in the middle that intersects no other interval. Any vertices omitted by $F$ are assigned intervals that intersect no others.

For the remaining forests, choose a root in each component. For each non-root vertex $v$, let $u$ be its neighbor along the path to the root, and introduce a small interval for $v$ inside the displayed portion of the interval for $u$ in the representation for $F$. For each vertex, we use at most one interval for each forest in the decomposition. 
With this tool, most bounds in Theorem 1.4 follow from known results. Proving $b(G) \leq 2$ when $G$ is an outerplanar digraph is more difficult and will be done in the next section.

Corollary 2.3. Let $G$ be an oriented graph. If $\hat{G}$ is planar, then $b(G) \leq 4$, and $b(G) \leq 3$ if $\hat{G}$ is also triangle-free or contains no subdivision of $K_{2,3}$. If $\hat{G}$ is planar and has girth at least 6 , then $b(G) \leq i_{2}(\hat{G}) \leq 2$.

Proof. Since every forest has fewer edges than vertices, always $\Upsilon(H) \geq \max _{F \subseteq H}\left[\frac{|E(F)|}{|V(F)-1|}\right]$, and Nash-Williams [22] proved that equality always holds. By Euler's Formula, it thus follows that the arboricity is at most 3 for every planar graph and is at most 2 for every triangle-free planar graph and every outerplanar graph. Balogh et al. [1] showed that a planar graph with no subdivision of $K_{2,3}$ has arboricity at most 2 .

When $G$ is planar and $\hat{G}$ has girth at least 6 , the upper bound improves from 3 to 2 by decomposing $\hat{G}$ into a forest and a linear forest, as guaranteed by Kleitman [18].

Kleitman's result is unpublished but follows from other results about forest decomposition. The fractional arboricity of a graph $H$, introduced by Payan [24] and denoted by $\operatorname{Arb}(H)$, is $\max _{F \subseteq H} \frac{|E(F)|}{|V(F)|-1}$; thus $\lceil\operatorname{Arb}(H)\rceil=\Upsilon(H)$. When $\operatorname{Arb}(H)$ is slightly more than $k$, decomposition needs $k+1$ forests, but some control can be exercised over one of the forests. Montassier et al. [21] posed the Nine Dragon Tree Conjecture: if $\operatorname{Arb}(H) \leq k+\frac{d}{k+d+1}$, then $H$ decomposes into a forest with maximum degree at most $d$ and $k$ other forests. They proved this for $k=1$ when $d \in\{1,2\}$. In [16], it was proved also for $d=k+1$ and for $k=1$ when $d \leq 6$.

Here the relevant case is $d=2$. By Euler's Formula, $\operatorname{Arb}(H) \leq \frac{g}{g-2}$ when $H$ is a planar graph with girth at least $g$. With $g=6, \frac{g}{g-2}=1+\frac{2}{4}$. Thus every planar graph $H$ with girth at least 6 decomposes into a linear forest and one other forest; that is, $\Upsilon^{\prime}(G) \leq 2$ when $G$ is an orientation of $H$. Since the Nine Dragon Tree Conjecture is true for $(k, d)=(1,2)$, the result applies to a larger class not requiring planarity.

Corollary 2.4. If $\operatorname{Arb}(H) \leq \frac{3}{2}$ and $G$ is an orientation of $H$, then $b(G) \leq 2$.

It remains open whether there are planar digraphs with visibility number 4 . We show next that the upper bound in Theorem 1.4 for triangle-free planar graphs is sharp.

Proposition 2.5. If $G$ is an orientation of a bipartite graph $H$, then $b(G) \leq i(H)$, with equality when all edges are oriented from one partite set to the other.

Proof. Since $H$ is triangle-free, every multi-interval representation of it has depth 2, and Proposition 2.1 applies to yield $b(G) \leq i(H)$.

When every vertex in $G$ is a source or a sink, a $k$-fold layout must have depth 2 . Projecting the bars onto a single horizontal line then yields a $k$-interval representation. 
Corollary 2.6. The largest visibility number among triangle-free planar digraphs is 3 .

Proof. Let $H$ be the graph obtained by appending a leaf to each vertex in the large partite set of the complete bipartite graph $K_{2,9}$; note that $H$ is triangle-free, planar, and bipartite.

As shown in [26], $i(H)=3$. Only eight of the pendant edges can be represented using intervals for the large part that contain endpoints of intervals for the small part; the higher-degree endpoint of the remaining pendant edge must be assigned three intervals. By Proposition 2.5, an orientation of $H$ directing all edges from one partite set to the other has visibility number 3 .

Among orientations of a bipartite graph, those with largest visibility number direct all edges from one partite set to the other, by Proposition 2.5. It is natural to seek the least visibility number among all orientations of a graph $H$. We do not define a separate parameter for this, since usually it equals the visibility number of $H$. As remarked in the introduction, the visibility number of $H$ is a lower bound on the visibility number of any orientation, but it is not clear that equality is always achievable.

For example, consider complete bipartite graphs. Trotter and Harary [28] proved that $i\left(K_{m, n}\right)=\left\lceil\frac{m n+1}{m+n}\right\rceil$, so this is the largest visibility number among orientations of $K_{m, n}$. By Theorem 1.7, $\left\lceil\frac{m n+4}{2 m+2 n}\right\rceil$ is a lower bound; let this value be $r$. Chang et al. [7] proved that the visibility number of $K_{m, n}$ is $r$ or $r+1$. Cao [6] improved the result to the exact answer: $r$ is always achievable. Achieving the counting bound when $\frac{m n+4}{2 m+2 n}$ is an integer forbids "redundancy" of representing an edge by multiple pairs of bars. In particular, bars for one vertex cannot see those for another both above and below, which implies in turn that there is an orientation of $K_{m, n}$ with visibility number $r$ (when $\frac{m n+4}{2 m+2 n}$ is an integer).

Another bipartite example is the $d$-dimensional hypercube. By Theorem 1.8, the upper bound on the visibility number of orientations is $\lceil(d+1) / 2\rceil$. By Theorem 1.7, the lower bound is $\lceil(d+1) / 4\rceil$. It is not known whether the visibility of the undirected hypercube always achieves the lower bound $\lceil(d+1) / 4\rceil$; if so, then again when $d \equiv 3 \bmod 4$ there can be no redundancy, and there will be an orientation achieving the lower bound. Equality has been proved for $d \leq 10$ [19]. These examples suggest a general conjecture.

Conjecture 2.7. The visibility number of any digraph is at most twice the visibility number of its underlying graph.

Oriented linear forests are not the only oriented forests with visibility number 1 . When $G$ has a smallest forest decomposition using oriented forests with visibility number 1 that are not linear forests, Observation 1.6 reduces the upper bound from $1+\Upsilon(G)$ to $\Upsilon(G)$ in a different way. A caterpillar is a tree that becomes a path when all leaves are deleted. Since caterpillars are bipartite interval graphs, the same argument as in Proposition 2.1 implies that caterpillars are bar-visibility digraphs. 
Corollary 2.8. If $G$ is an oriented graph, and $\hat{G}$ decomposes into $\Upsilon(\hat{G})$ caterpillars, then $b(G) \leq \Upsilon(\hat{G})$.

Proof. Proposition 2.1 and Observation 1.6.

The caterpillar arboricity of a graph $H$, written ca $(H)$, is the minimum number of forests of caterpillars needed to decompose $H$. Every outerplanar graph decomposes into two forests, but some have caterpillar arboricity 3. Kostochka and West [20] constructed such a graph $H$ as follows: Let $K_{4}^{-}$be the graph with four vertices and five edges. Let $H^{\prime}$ be the union of three copies of $K_{4}^{-}$sharing a common vertex $x$ that has degree 2 in each copy. Let $H$ consist of five disjoint copies of $H^{\prime}$ plus one new vertex $w$ adjacent to the five copies of $x$.

In fact, infinitely many 2-connected outerplanar graphs have caterpillar arboricity 3 . If $H_{k}$ is the outerplanar graph whose weak dual consists of a vertex of degree 3 whose neighbors are the roots of three complete binary trees of depth $k-1$, then ca $\left(H_{k}\right)=3$ for large enough $k$. We omit the details.

However, in Section 3 we will prove that every outerplanar digraph has visibility number at most 2 . We close this section by proving the result on trees stated in the introduction.

(Proposition 1.3). If $G$ is an oriented tree, then $b(G) \leq 2$, with equality if and only if $G$ has a vertex that is an endpoint of three edge-disjoint inconsistent paths.

Proof. Every tree has caterpillar arboricity at most 2. In fact, every tree $T$ decomposes into two forests of stars: root $T$ at some vertex $v$, let one subgraph contain all edges joining vertices at distance $2 l$ and $2 l+1$ from $v$ (for $l \geq 0$ ), and let the other subgraph contain the remaining edges. By Corollary 2.8, $b(G) \leq 2$ when $G$ is an oriented tree.

A vertex $v$ in an oriented tree $G$ is problematic if three edge-disjoint inconsistent paths end at it. We prove that $b(G)=2$ if $G$ has a problematic vertex; otherwise $b(G)=1$.

Suppose first that $G$ has a problematic vertex $v$ and has a single-bar layout. Since the $v$-bar has only two endpoints, it must see the entire bar assigned to its neighbor on one of the three inconsistent paths; call that path $P$. Let $x, y, z$ be the three consecutive vertices along $P$ such that $y$ is the first source or sink encountered along $P$ after starting from $v$. While following $P$ away from $v$, the bar for each successive vertex must be seen completely by the previous vertex until $y$ is reached. Now there is no way to assign a $z$-bar to see the $y$-bar in the correct direction without also seeing the $x$-bar.

Now suppose that $G$ has no problematic vertex. An inductive proof that $b(G)=1$ can be given using the characterization of visibility digraphs in Theorem 1.2. Instead, we give a short, self-contained proof. Given a path $P$ in $G$, we say a path $P^{\prime}$ in $G$ departs $P$ if it is arc-disjoint from $P$ and has one endpoint in $P$. We claim that $G$ contains a path $P$ such that all paths departing $P$ are consistent. 
Among all oriented paths in $G$, let $P$ be one with the fewest inconsistent departing paths. Suppose that $Q$ is an inconsistent path departing $P$ at a vertex $v$. Let $P_{1}$ and $P_{2}$ be the two oriented paths whose union is $P$ and whose intersection is the vertex $v$. Since $v$ is not problematic, all inconsistent paths departing $P$ other than $Q$ must depart via one of $\left\{P_{1}, P_{2}\right\}$; without loss of generality, all inconsistent paths departing $P$ have endpoints in $P_{2}$. Now the path formed from $P$ by replacing $P_{1}$ with $Q$ has fewer inconsistent departing paths, which contradicts the choice of $P$.

With $P$ so chosen, create a single-bar layout for $P$, with each bar having a portion that sees no other bar above or below it. Each component of $G-V(P)$, together with the arc joining it to a vertex $v$ of $P$, is a rooted tree directed outward from $v$ or inward to $v$. Each has a single-bar layout that can be placed in the region above or below a portion of the $v$-bar seeing nothing above or below it. These additions to the layout can be placed independently to complete a single-bar layout of $G$.

\section{Outerplanar digraphs}

In this section we prove that outerplanar digraphs have visibility number at most 2. Outerplanar graphs have interval number at most 2, since the 3-interval representations of planar graphs produced in [26] use at most two intervals for each vertex on the unbounded face. Unfortunately, those representations do not have depth 2. Also, as remarked in Section 2, some outerplanar graphs have caterpillar arboricity 3. Hence we need a direct proof that $b(G) \leq 2$ when $G$ is an outerplanar digraph.

In the proof of Proposition 1.3, we used the notion of empty "corridors" above or below part of a bar. We next formalize and refine this notion. In these definitions, the endpoints of intervals and bars are not important, because lines of sight require positive width.

Definition 3.1. In a visibility layout, an up-corridor is a set of the form $I \times(y, \infty)$ that intersects no bar, where $I$ is an interval of positive length. Similarly, a down-corridor is a set of the form $I \times(-\infty, y)$ with those properties. A segment of a bar is a connected subset of it with positive length. A segment $I \times\{y\}$ is up-displayed if $I \times(y, \infty)$ is an up-corridor, and it is down-displayed if $I \times(-\infty, y)$ is a down-corridor. For a vertex $x$, let $U_{x}$ denote an up-corridor above an up-displayed segment of an $x$-bar, and let $D_{x}$ denote a down-corridor below a down-displayed segment of an $x$-bar, when such corridors exist.

Laying out a linear forest as in Proposition 2.2 gives each vertex both an up-corridor and a down-corridor. However, a bar subsequently inserted into an up-corridor will not be down-displayed, and vice versa. We next extend the notion of corridors to apply also to 
edges. For an outerplanar graph, the edges of the unbounded face are external edges; the others are internal edges. In an orientation, these become external arcs and internal arcs.

Definition 3.2. Consider a visibility layout. For adjacent vertices $x$ and $y$, an above-corridor $A_{x y}$ is an up-corridor that is the union of up-corridors $U_{x}$ and $U_{y}$ sharing a vertical boundary; $U_{x}$ is above an up-displayed segment of an $x$-bar, and $U_{y}$ is above an up-displayed segment of a $y$-bar. Similarly, a below-corridor $B_{x y}$ is a down-corridor that is the union of down-corridors $D_{x}$ and $D_{y}$ sharing a vertical boundary. See Figure 3.1.

When we number the vertices as $v_{1}, \ldots, v_{n}$, we use only $i$ and $j$ in the subscripts naming corridors, rather than $v_{i}$ and $v_{j}$. Note that for an above-corridor $A_{x y}$, the higher of the $x$-bar and $y$-bar has an endpoint whose horizontal coordinate is that of the common vertical boundary of the up-corridors $U_{x}$ and $U_{y}$ whose union is $A_{x y}$ (similarly for down-corridors).

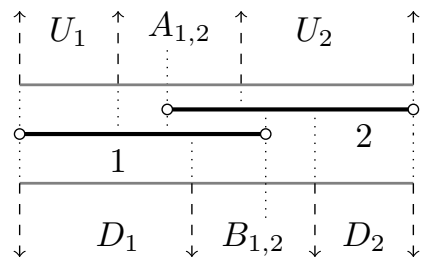

Figure 3.1: Corridors in a layout for a graph having an arc from $v_{1}$ to $v_{2}$.

Definition 3.3. For an interval $I$ of positive length, the set $I \times(-\infty, \infty)$ is an open-corridor if it intersects exactly one bar $b$ in the layout, and $b$ has exactly one endpoint with horizontal coordinate internal to $I$. We then say that $b$ is doubly-displayed. A full-corridor $F_{x}^{y}$ is an open-corridor for an $x$-bar such that $x$ and $y$ are the endpoints of an external arc. A fullcorridor for an $x$-bar can only be named for one external neighbor $y$ of $x$. See Figure 3.2.
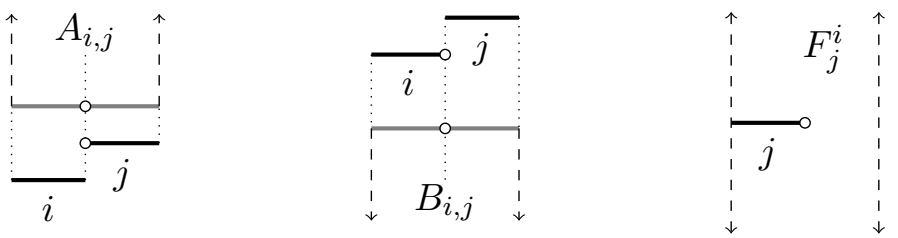

Figure 3.2: Corridors for an external arc from $v_{i}$ to $v_{j}$.

(Theorem 1.5). If $G$ is an outerplanar digraph, then $b(G) \leq 2$.

Proof. We may assume that $\hat{G}$ is connected, since otherwise we can combine layouts for the components of $G$. We first prove the bound when $\hat{G}$ is 2 -connected. Later, we explain how 
to combine layouts of such digraphs when $\hat{G}$ is not 2-connected. In each case, we prove a stronger statement about outerplanar digraphs, showing that $G$ has a 2 -fold layout in which the bars corresponding to the endpoints of external arcs satisfy additional requirements.

Given an outerplane embedding $H$ of a 2-connected planar graph, form a special "eardecomposition" $P_{1}, \ldots, P_{r}$ as follows. First, let $P_{1}$ be any external edge. For $k>1$, let $P_{k}$ be a path that completes a face in $H$ with an external edge of the subgraph $H_{k}$ formed by $P_{1} \cup \cdots \cup P_{k-1}$. With multi-edges forbidden, each $P_{k}$ has length at least 2 , and $H_{k}$ is a 2-connected outerplanar graph. Let $n_{k}=\left|V\left(H_{k}\right)\right|$ and label $V(H)$ and $\left\{v_{1}, \ldots, v_{n}\right\}$ so that $V\left(P_{1}\right)=\left\{v_{1}, v_{2}\right\}$, and for $k>1$ the internal vertices of $P_{k}$ are $v_{n_{k-1}+1}, \ldots, v_{n_{k}}$.

Given an outerplanar digraph $G$, let $\mathcal{E}$ be a subset of its set of external arcs. An $\mathcal{E}$-special layout of $G$ is a double-bar layout such that the following hold:

(1) For $v_{i} \in V(G)$, there is an up-corridor $U_{i}$ and a down-corridor $D_{i}$.

(2) For an arc in $\mathcal{E}$ on $\left\{v_{i}, v_{j}\right\}$ with $i<j$, a set $\mathcal{C}_{i, j}$ is assigned consisting of two among $\left\{A_{i, j}, B_{i, j}, F_{j}^{i}\right\}$ (corridors of two types among above-, below-, and full-).

(3) All corridors specified in (1) and (2) are disjoint.

Given the decomposition $P_{1}, \ldots, P_{r}$ of $\hat{G}$, let $G_{k}$ be the union of the orientations of $P_{1}, \ldots, P_{k}$ in $G$. Let $\mathcal{E}_{1}$ be the arc in $G_{1}$. For $k \geq 2$, let $\mathcal{E}_{k}$ be the set of external arcs in $G_{k}$ other than that in $\mathcal{E}_{1}$. We prove by induction on $r$ that $G$ has an $\mathcal{E}_{r}$-special layout.

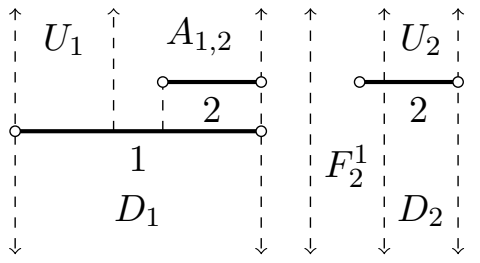

(a)

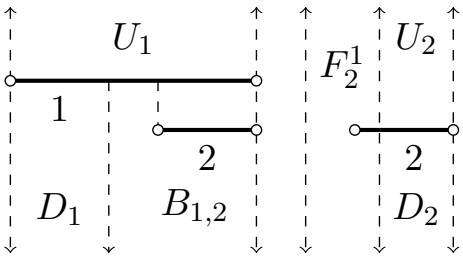

(b)

Figure 3.3: $G_{1}$ : (a) $\operatorname{arc} v_{1} v_{2}$ with $\mathcal{C}_{1,2}=\left\{A_{1,2}, F_{2}^{1}\right\}$, (b) arc $v_{2} v_{1}$ with $\mathcal{C}_{1,2}=\left\{B_{1,2}, F_{2}^{1}\right\}$.

For $r=1$, we have the degenerate case of a single arc (not 2-connected). Depending on whether the arc is $v_{1} v_{2}$ or $v_{2} v_{1}$, use one of the double-bar layouts in Figure 3.3. The requirements for an $\mathcal{E}_{1}$-special layout are satisfied.

It suffices to obtain an $\mathcal{E}_{r}$-special layout of $G_{r}$, given an $\mathcal{E}_{r-1}$-special layout of $G_{r-1}$. Let $v_{i}$ and $v_{j}$ with $i<j \leq n_{r-1}$ be the endpoints of $P_{r}$. The arc with endpoints $v_{i}$ and $v_{j}$ belongs to $\mathcal{E}_{r-1}$, and $\left\{v_{i}, v_{j}\right\}=\left\{v_{1}, v_{2}\right\}$ if and only if $r=2$.

If $n_{r}-n_{r-1}=1$, then the added vertex $v_{k}$ (where $k=n_{r}$ ) creates a triangle with $v_{i}$ and $v_{j}$. There are twelve cases, depending on which are the two types of corridors in $\mathcal{C}_{i, j}$ and how the two new $\operatorname{arcs}$ (on $\left\{v_{i}, v_{k}\right\}$ and $\left\{v_{j}, v_{k}\right\}$ ) are oriented. Layouts for the two added 
$v_{k}$-bars are shown in Figure 3.4 for $\mathcal{C}_{i, j}=\left\{A_{i, j}, B_{i, j}\right\}$, in Figure 3.5 for $\mathcal{C}_{i, j}=\left\{A_{i, j}, F_{j}^{i}\right\}$, and in Figure 3.6 for $\mathcal{C}_{i, j}=\left\{B_{i, j}, F_{j}^{i}\right\}$.

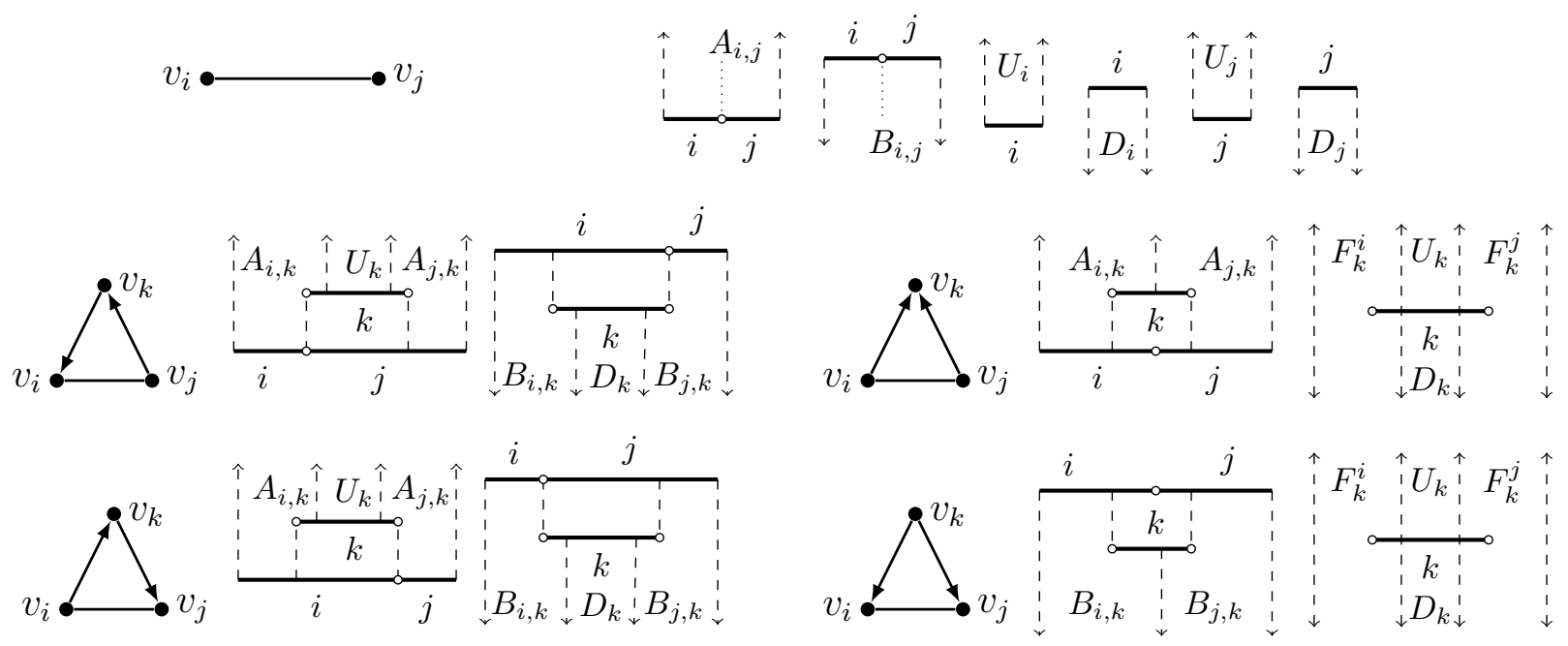

Figure 3.4: The case $\mathcal{C}_{i, j}=\left\{A_{i, j}, B_{i, j}\right\}$

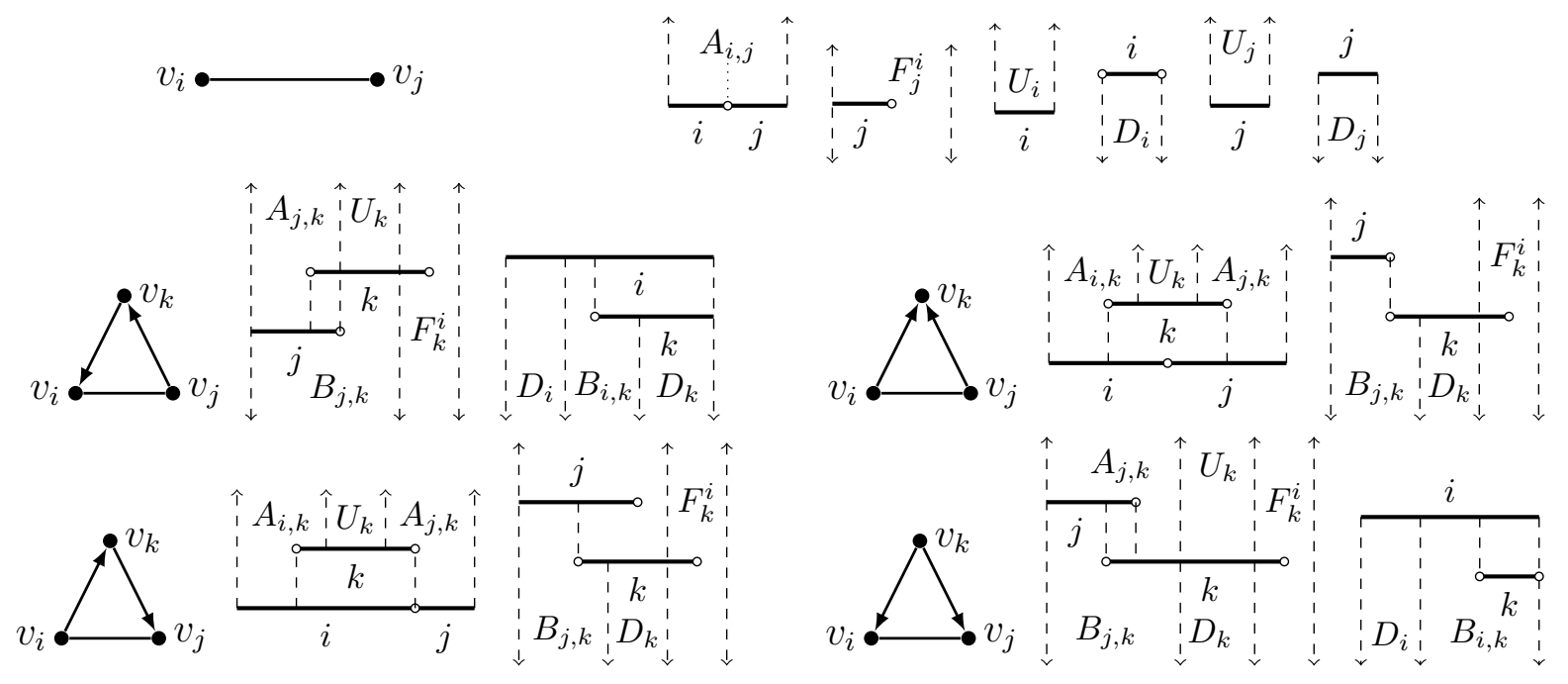

Figure 3.5: The case $\mathcal{C}_{i, j}=\left\{A_{i, j}, F_{j}^{i}\right\}$.

Each figure lists the available corridors at the top before adding bars for $v_{k}$. Below that, the four cases for orienting the arcs on $\left\{v_{i}, v_{k}\right\}$ and $\left\{v_{j}, v_{k}\right\}$ appear, together with the updates 


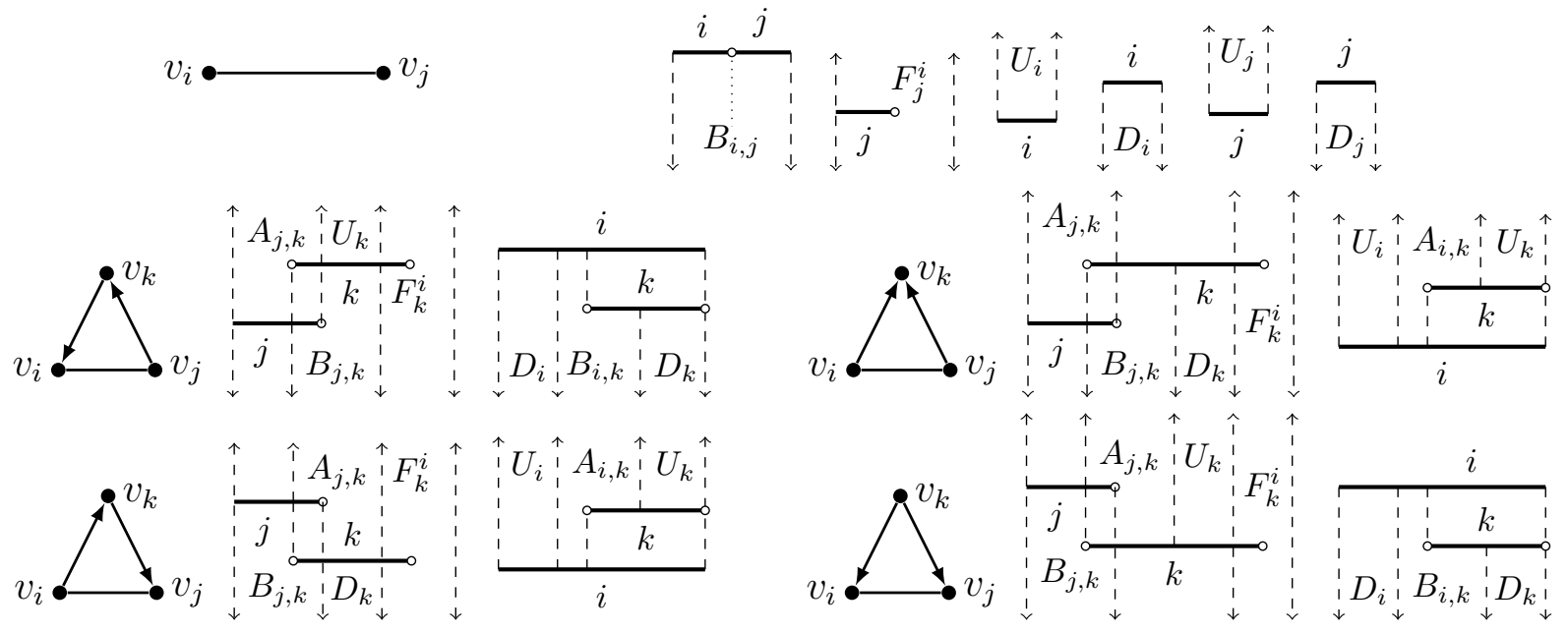

Figure 3.6: The case $\mathcal{C}_{i, j}=\left\{B_{i, j}, F_{j}^{i}\right\}$.

to the corridors. Only the altered corridors are shown. The requirement for $\mathcal{C}_{i, j}$ is dropped, because the arc on $\left\{v_{i}, v_{j}\right\}$ is no longer external, the up- and down-corridors for $v_{i}$ and $v_{j}$ are preserved, the up- and down-corridors for $v_{k}$ are introduced, and the requirements for $\mathcal{C}_{i, k}$ and $\mathcal{C}_{j, k}$ are introduced. Thus in each case the resulting layout is $\mathcal{E}_{r}$-special. We often stretch the layout horizontally when building onto an existing full-corridor $F_{j}^{i}$.

Note that corridor $F_{j}^{i}$ exclusively claims an end of an existing $j$-bar, which can only be used (and hence lost) when creating a new face using the arc on $\{i, j\}$. This systematic reservation of full-corridors is essential to the success of the layout. It also explains why the arc on $\left\{v_{1}, v_{2}\right\}$ is excluded from $\mathcal{E}_{r}$ for $r>1$; although the arc remains external, there is no need and no ability to preserve the full-corridor $F_{2}^{1}$ when $v_{3}$ is added.

If $n_{r}-n_{r-1}>1$, then create a single-bar layout for the oriented path $v_{n_{r-1}+1}, \ldots, v_{n_{r}}$ (as in the proof of Proposition 2.1), independent from the layout of $G_{r-1}$. This layout introduces corridors $U_{i}$ and $D_{i}$ for $n_{r-1}+1 \leq i \leq n_{r}$, as well as $A_{i, i+1}$ and $B_{i, i+1}$ for $n_{r-1}+1 \leq i \leq n_{r}-1$. It remains to represent the arcs on $\left\{v_{i}, v_{n_{r-1}+1}\right\}$ and $\left\{v_{j}, v_{n_{r}}\right\}$ and give them visibility corridors.

This case is easier than $n_{r}-n_{r-1}=1$ because now $v_{n_{r-1}+1}$ and $v_{n_{r}}$ are distinct vertices. Let $k=n_{r-1}+1$, and consider the arc on $\left\{v_{i}, v_{k}\right\}$. We created only one $v_{k}$-bar in representing the path and have another available. Depending on the orientation of $\left\{v_{i}, v_{k}\right\}$, place a $v_{k}$-bar in $U_{i}$ or $D_{i}$ to create $A_{i, k}$ or $B_{i, k}$ and to maintain (a shrunken) $U_{i}$ or $D_{i}$. Finally, use the end of the $v_{k}$-bar in the path layout to create $F_{k}^{i}$. After handling both $\left\{v_{i}, v_{n_{r-1}+1}\right\}$ and $\left\{v_{j}, v_{n_{r}}\right\}$ in this manner, we have an $\mathcal{E}_{r}$-special layout. This completes the induction. 
Now let $G$ be an arbitrary connected outerplanar digraph. Let $G_{1}, \ldots, G_{\ell}$ be the orientations of the blocks of $\hat{G}$ that are subgraphs of $G$, ordered so that $\bigcup_{j=1}^{i} \hat{G}_{j}$ is connected for $1 \leq i \leq \ell$. Again we prove a stronger statement about the layout by induction on $\ell$. Say that a double-bar layout of an outerplanar digraph $G$ is vertex-special if for every $v_{i} \in V(G)$, there is an up-corridor $U_{i}$ and a down-corridor $D_{i}$, and all specified corridors are disjoint.

We create a vertex-special layout for $G$. An $\mathcal{E}$-special layout of a 2-connected outerplanar graph is vertex-special, so the layout exists when $\ell=1$. For $\ell>1$, let $G^{\prime}=\bigcup_{j=1}^{\ell-1} G_{j}$, and obtain a vertex-special layout for $G^{\prime}$ from the induction hypothesis. Let $v_{1}$ be the vertex shared by $G^{\prime}$ and $G_{\ell}$. Choose $v_{2} \in V\left(G_{\ell}\right)$ so that the $\operatorname{arc}$ on $\left\{v_{1}, v_{2}\right\}$ is external in $G_{\ell}$. Add $v_{2}$-bars as in Figure 3.3 to represent this arc, placing a $v_{2}$-bar inside the existing corridor $U_{1}$ or $D_{1}$ for $v_{1}$ to create $A_{1,2}$ or $B_{1,2}$. Claim a full-corridor $F_{2}^{1}$ around one endpoint of the other $v_{2}$-bar. Lay out the rest of $G_{\ell}$ using the procedure described above for a 2-connected outerplanar digraph. The resulting layout of $G$ is vertex-special.

Note that in the final induction, we do not have the choice of which vertex is $v_{1}$. This explains why we need both alternatives in Figure 3.3. Figure 3.7 shows a maximal outerplanar digraph and the double-bar layout resulting from the proof.
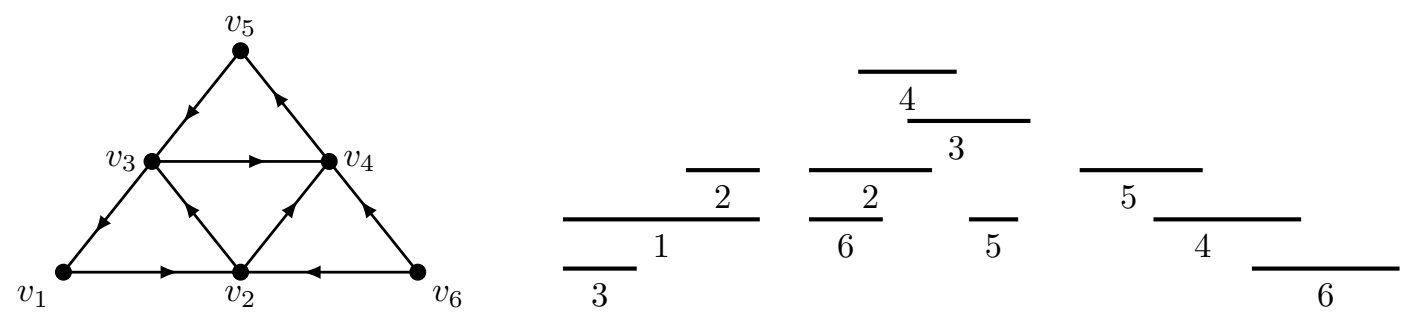

Figure 3.7: A maximal outerplanar digraph and its double-bar layout.

\section{General oriented graphs}

In this section, we prove Theorem 1.9. Our layouts use Steiner systems (cf. [4]). A Steiner system $S(2, k, m)$ is a family of $k$-subsets of an $m$-set $S$, called blocks or $k$-blocks, such that any two elements appear together in exactly one block. Such a family has exactly $\left(\begin{array}{c}m \\ 2\end{array}\right) /\left(\begin{array}{c}k \\ 2\end{array}\right)$ blocks, and each element appears in exactly $(m-1) /(k-1)$ blocks. Thus existence requires the following divisibility conditions:

$$
\begin{array}{rll}
m-1 & \equiv 0 \quad(\bmod (k-1)), \\
m(m-1) & \equiv 0 \quad(\bmod k(k-1)) .
\end{array}
$$


Determining when these conditions are sufficient is a central problem in the study of Steiner systems. Our arguments use the existence of $S(2,4, m)$ and $S(2,15, m)$. Hanani [11] proved that the divisibility conditions are sufficient when $k=4$.

Theorem 4.1 (Hanani [11]). When $k=4$, the necessary conditions of (1) are sufficient for the existence of $S(2, k, m)$. In particular, a Steiner system $S(2,4, m)$ exists when $m=12 s+1$ for $s \geq 1$ and when $m=12 s+4$ for $s \geq 0$.

For a survey of results on $S(2,4, m)$ Steiner systems, see [25]. For general $k$, Wilson [30] resolved the question when $m$ is sufficiently large in terms of $k$.

Theorem 4.2 (Wilson [30]). Let $k$ be a fixed integer. When $m$ is sufficiently large, the necessary conditions of (1) suffice for the existence of $S(2, k, m)$. In particular, there exists $c_{k}$ such that for $m \geq c_{k}$, a Steiner system $S(2, k, m)$ exists when $m=s k(k-1)+1$ and when $m=s k(k-1)+k$.

We begin with visibility layouts for all oriented graphs having at most four vertices (up to isomorphism). These are building blocks for the proof of Theorem 1.9. A bar has left reach (right reach) if its left (right) endpoint is leftmost (rightmost) in the layout. A vertex $v$ has full reach if some $v$-bar has left reach and some $v$-bar has right reach (possibly the same bar). Each layout in Figures 4.1-4.6 is a single-bar layout or is a double-bar layout with one vertex $v$ having two bars. In the latter case, $v$ has full reach. In our figures, left or right reach of a bar is shown by an arrowhead at the appropriate endpoint, and visibility between bars is indicated by vertical gray segments.

Trivially, oriented graphs with at most two vertices have single-bar layouts, as do 3vertex oriented graphs with fewer than three edges. As shown in Figure 4.1, a transitive 3-tournament has a single-bar layout, but a non-transitive 3-tournament (a consistent 3cycle) requires a double-bar layout. In each layout, we give full reach to a $v_{1}$-bar.
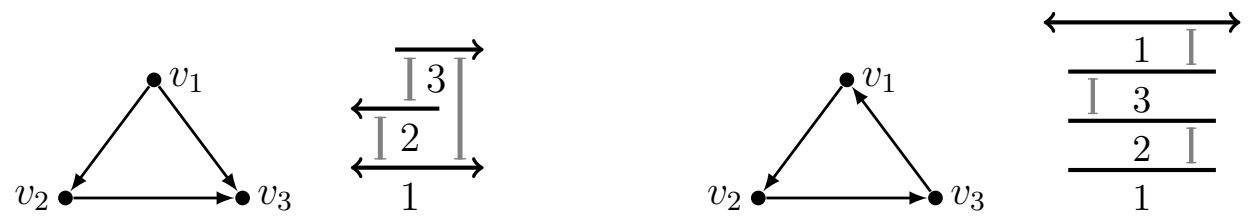

Figure 4.1: Layouts of 3-tournaments.

Next, let $G$ be an oriented graph with four vertices. If $|E(G)|<3$, then $G$ has a singlebar layout. If $|E(G)|=3$, then either $G$ is a tree with $b(G)=1$ by Proposition 1.3 , or $G$ consists of an isolated vertex and a tournament on 3 vertices, which can be laid out using Figure 4.1 and an isolated bar for the isolated vertex. 

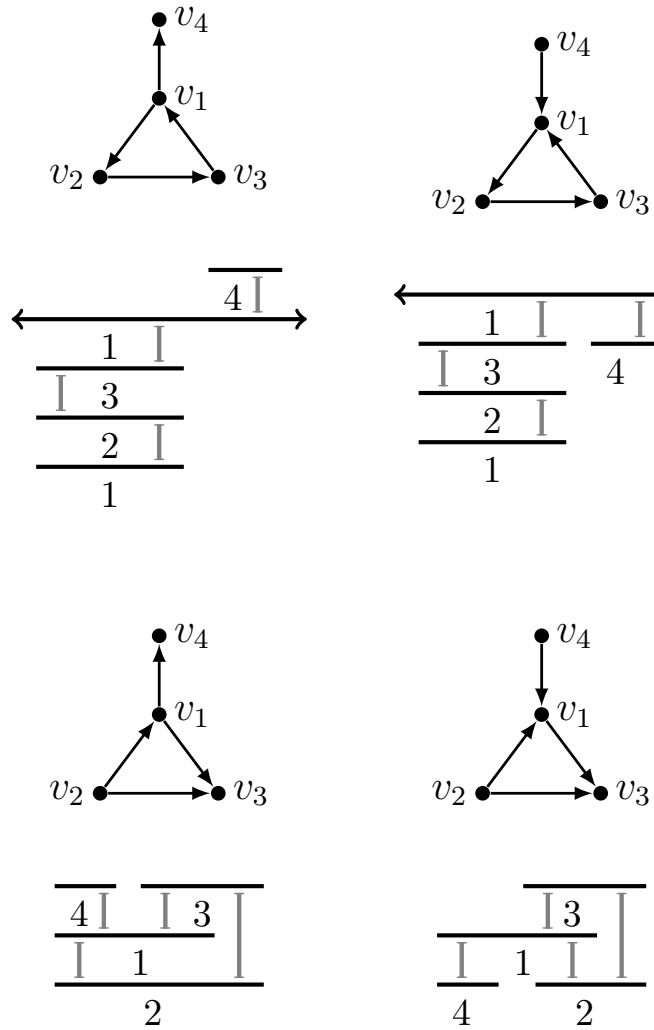
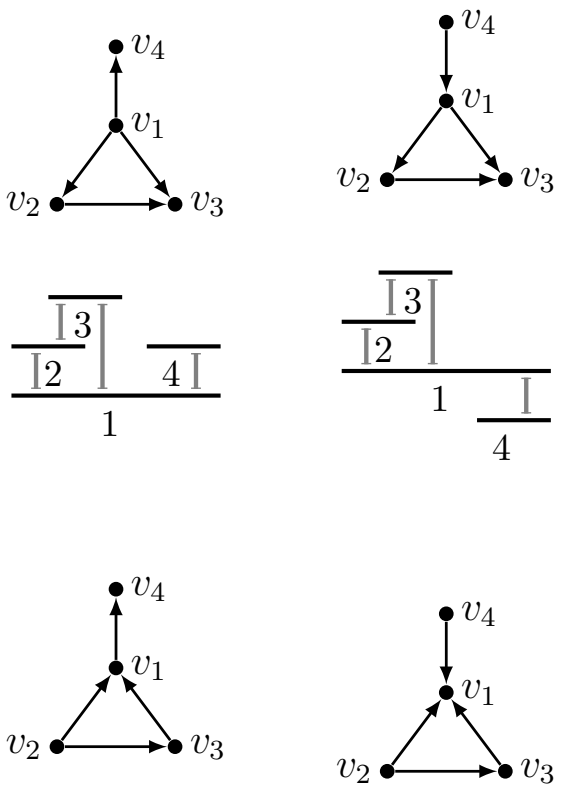

Figure 4.2: Layouts of orientations of a 3-cycle with a pendant edge.

If $|E(G)|=4$, then $\hat{G}$ is a 4-cycle or a 3-cycle plus a pendant edge. Figures 4.2 and 4.3 show all possible orientations (up to isomorphism) and corresponding visibility layouts. Figure 4.4 shows layouts of all possible orientations when $|E(G)|=5$.
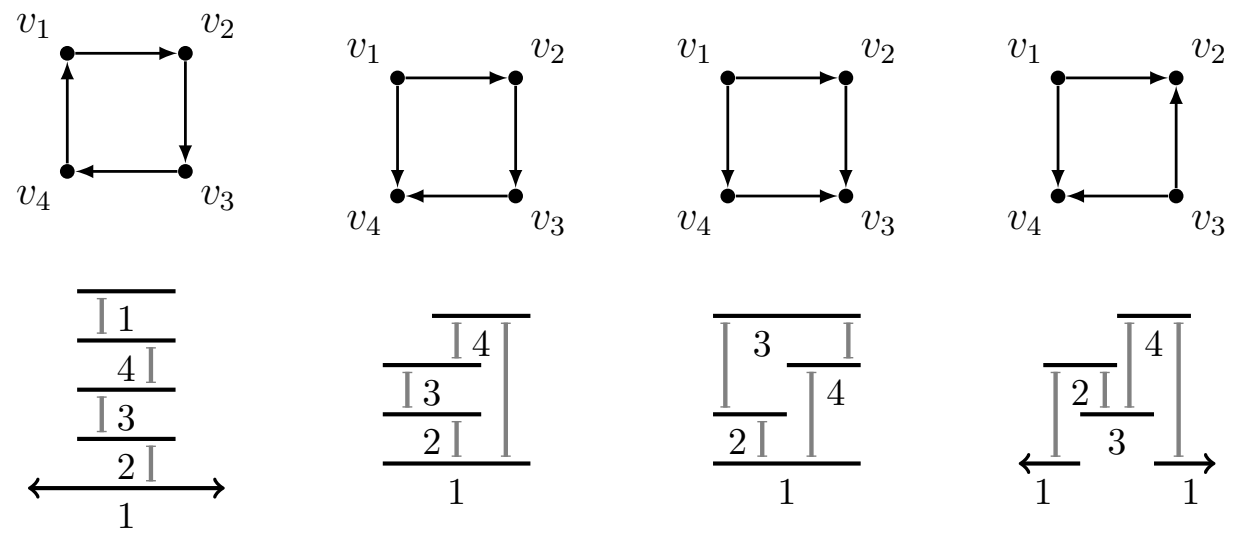

Figure 4.3: Layouts of oriented 4-cycles. 

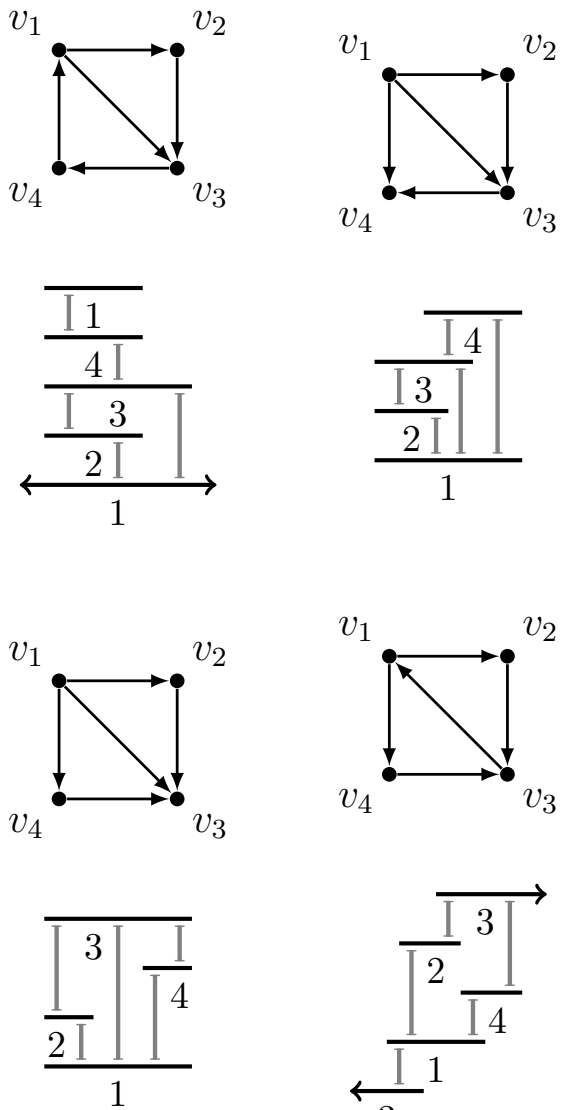
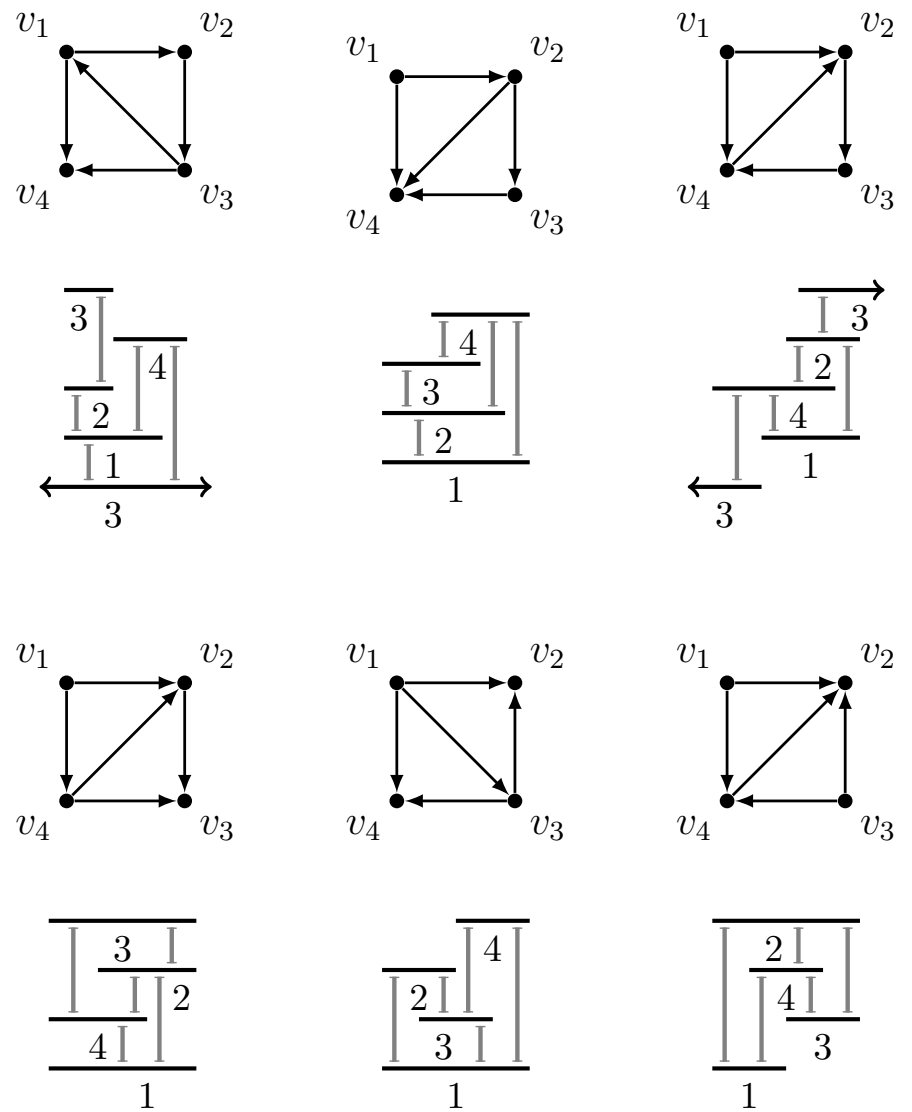

Figure 4.4: Layouts of digraphs with four vertices and five edges.

Finally, we consider 4-tournaments. A transitive 4-tournament has a single-bar layout. When the vertices are labeled so that $v_{i} v_{j} \in E(G)$ for $1 \leq i<j \leq 4$, we can give $v_{1}$ and $v_{4}$ full reach. Moreover, we can choose exactly one of $\left\{v_{2}, v_{3}\right\}$ to have left reach or right reach (but not both); see Figure 4.5. For all other 4-tournaments, we give a double-bar layout where the one vertex with two bars has full reach. Every non-transitive 4-tournament contains
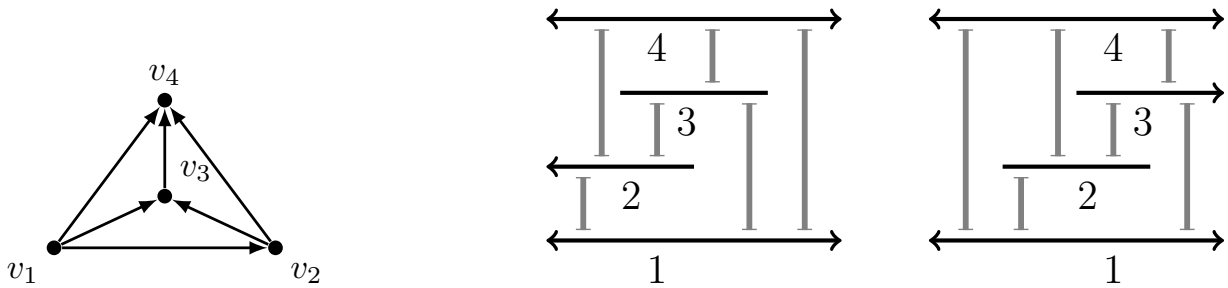

Figure 4.5: Single-bar layouts of a transitive 4-tournament. 

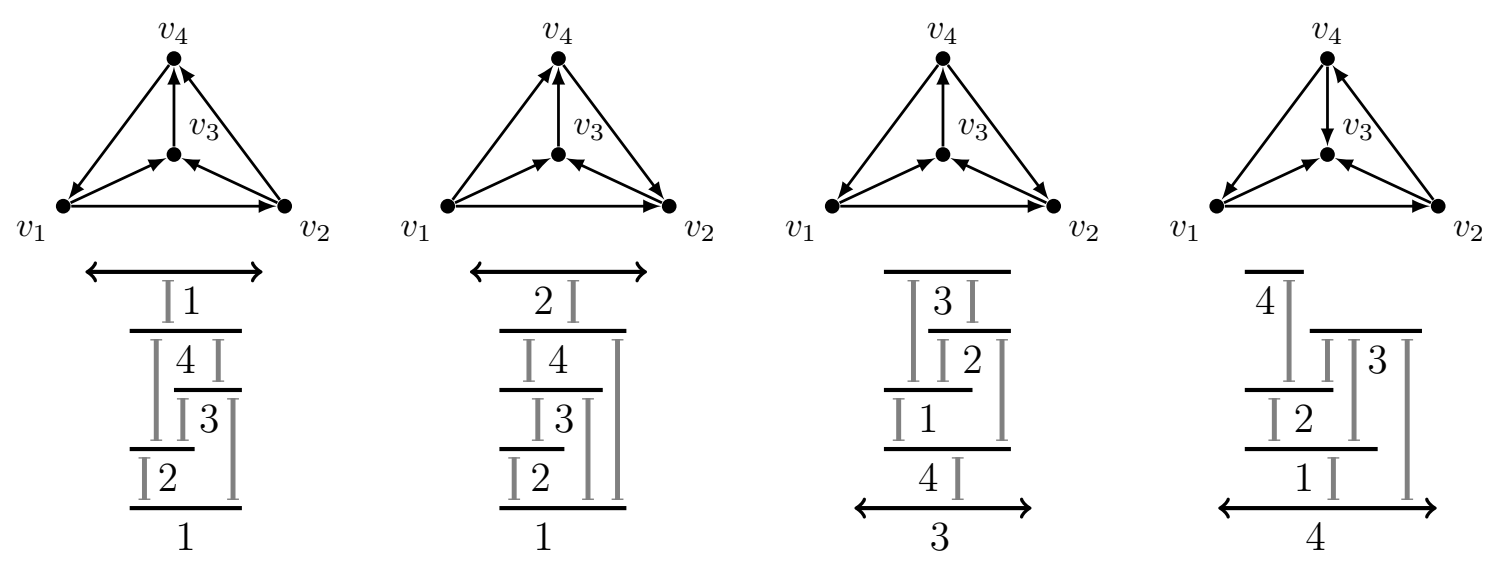

Figure 4.6: Visibility layouts for non-transitive 4-tournaments.

a transitive triple. Letting $\left\{v_{1}, v_{2}, v_{3}\right\}$ form such a triple (in order), there are four ways to orient the arcs incident with $v_{4}$ without creating a transitive 4-tournament. Figure 4.6 shows visibility layouts in which the one vertex with two bars has full reach.

(Theorem 1.9). If $G$ is a digraph with $n$ vertices, then $b(G) \leq(n+10) / 3$.

Proof. Let $V(G)=\left\{v_{i}: i \in[n]\right\}$. We use a Steiner system $S(2,4, m)$. Theorem 4.1 guarantees the existence of $S(2,4, m)$ for some $m$ with $n \leq m \leq n+8$. Add isolated vertices $\left\{v_{n+1}, \ldots, v_{m}\right\}$ to extend $G$ to a larger oriented graph $G^{\prime}$. Create a Steiner system $S(2,4, m)$ on $V\left(G^{\prime}\right)$; every vertex appears in $(m-1) / 3$ blocks. Create a new set system $S^{\prime}$ by deleting all instances of the extra $m-n$ vertices and deleting every resulting block of size 1 .

We now lay out $G$. For each block in $S^{\prime}$, lay out the corresponding induced subgraph using one of the layouts described in Figures 4.1-4.6. Blocks having single-bar layouts are left as components of the layout. Combine the blocks having two bars for a particular vertex $v$ into a single component of the layout as follows. In each such block, $v$ has full reach. Lay out one such block as the leftmost. Iteratively, adjust the vertical position of the next such block so that its $v$-bar with left reach has the same vertical coordinate as the $v$-bar with right reach in the current block. Extend these two bars to combine.

Having combined these blocks into one component of the layout, we now have one $v$ bar for each block containing $v$, plus one extra $v$-bar in the first block used in the large component for $v$. Since $m \leq n+8$ and each vertex appears in at most $(m-1) / 3$ blocks, each vertex is assigned at most $(n+10) / 3$ bars. 


\section{Transitive tournaments}

Up to isomorphism, there is only one transitive tournament on $n$ vertices, call it $T_{n}$. We label the vertices so that the arcs are $\left\{v_{i} v_{j}: i<j\right\}$. In layouts of $T_{n}$, we may assume that all $v_{k}$-bars have vertical coordinate $k$.

We begin by giving visibility layouts for $T_{n}$ with $n \leq 15$. The layout of $T_{n}$ will be used to prove the upper bound in Theorem 1.10(b) for large transitive tournaments. The layouts in Section 4 show that if $n \leq 4$, then $b\left(T_{n}\right)=1$. Since $K_{5}$ is not planar, $b\left(T_{5}\right) \geq 2$. Figure 5.1 gives a 2 -fold layout of $T_{10}$, which proves $T_{n}=2$ for $5 \leq n \leq 10$. Here we are using the key fact that removing bars from a layout of a transitive tournament cannot introduce any unwanted visibilities. Figure 5.2 gives a 3 -fold layout of $T_{15}$; thus $b\left(T_{n}\right) \leq 3$ for $11 \leq n \leq 15$. Equality holds at least for $13 \leq n \leq 15$ because the visibility number of $K_{n}$ is $\lceil n / 6\rceil$ [7].

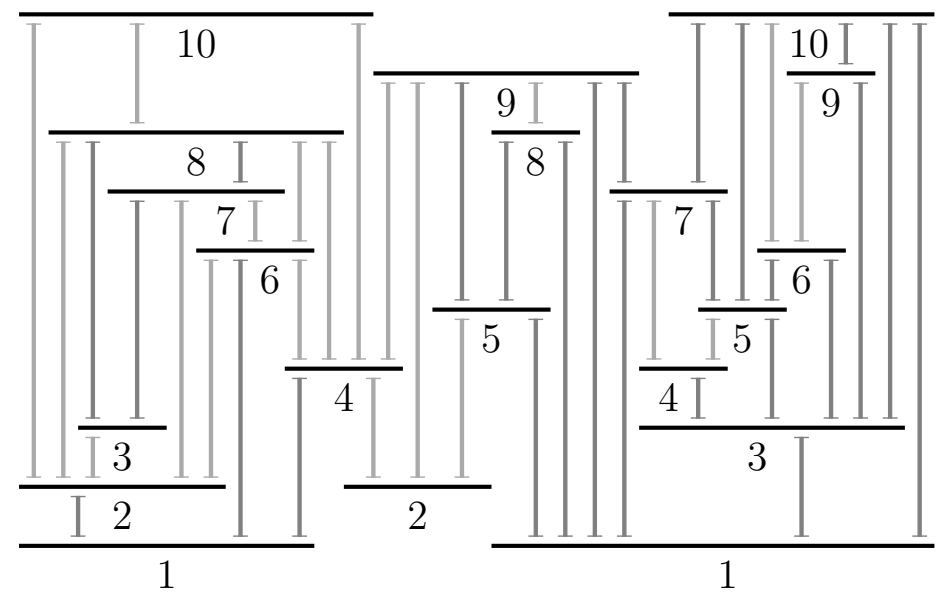

Figure 5.1: A 2-fold layout of the transitive 10-tournament.

Several open questions remain in this area. We conjecture that $b\left(T_{11}\right)=3$, yielding $b\left(T_{n}\right)=3$ for $11 \leq n \leq 15$. We ask whether $b\left(T_{16}\right) \leq 3$ and whether $b\left(T_{20}\right) \leq 4$. In general, is there a $k$-fold layout of the transitive $5 k$-tournament for some $k$ larger than 3 ? If this holds for all $k$, then the upper bound improves to $b\left(T_{n}\right) \leq\lceil n / 5\rceil$. If it holds for some $k$, then using $S(2,5 k, m)$ with $m$ a bit larger than $n$ yields $b\left(T_{n}\right) \leq \frac{k}{5 k-1} n+c$, where $c$ is a constant.

Theorem 1.9 yields $b(G) \leq(n+10) / 3$ for every $n$-vertex digraph $G$. For $T_{n}$, we reduce the leading coefficient. Again we use 4-vertex blocks, but we need to use fewer bars for each vertex $v$ than the number of blocks containing it. The key is that any two blocks containing $v$ can be combined into a single component of the layout using just one block for $v$.

(Theorem 1.10(a)). $b\left(T_{n}\right) \leq 7 n / 24+2 \sqrt{n \log n}$. 


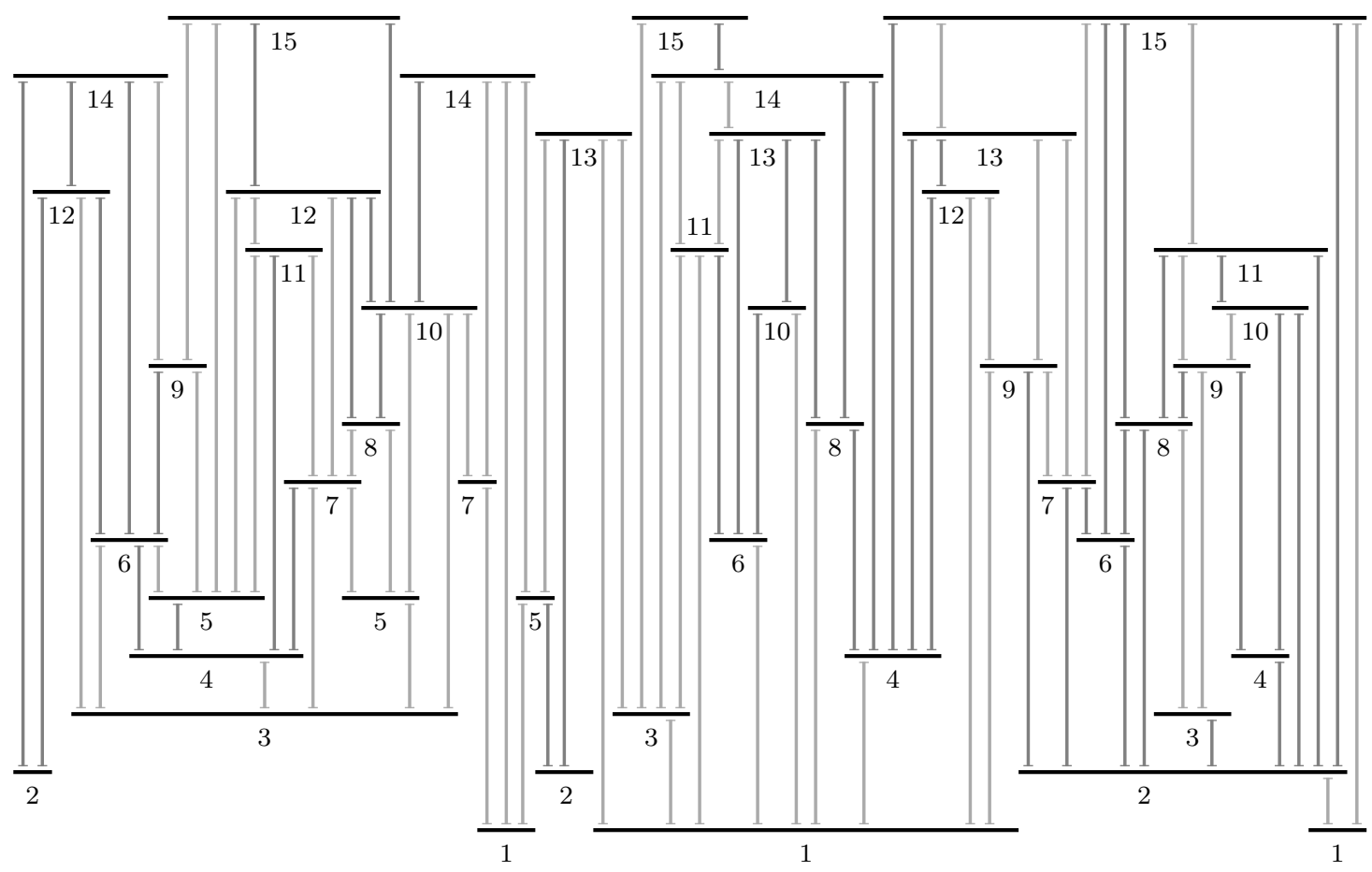

Figure 5.2: A 3-fold layout of a transitive 15-tournament

Proof. From the layouts in Figures 5.1 and 5.2, we may assume $n \geq 16$. As in Theorem 1.9, we use the Steiner system $S(2,4, m)$ with $n \leq m \leq n+8$ having added vertices $v_{n+1}, \ldots, v_{m}$ (all arcs oriented toward the higher-indexed endpoint). Since $b\left(T_{4}\right)=1$, independently laying out the subtournaments induced by the blocks yields a layout with $(m-1) / 3$ bars per vertex.

We use a probabilistic construction to combine components in this layout. As shown in Figure 4.5, $T_{4}$ has single-bar layouts such that any desired vertex is given left reach or right reach. By adjusting vertical coordinates, layouts of the subtournaments on two 4-element sets with a common vertex $v$ can be combined to lay out the union using only one bar for $v$. Call this combined layout a $v$-barbell.

The number of $v$-bars is now $(m-1) / 3$ minus the number of $v$-barbells. Hence we want to group the blocks into pairs to create many $v$-barbells for each vertex $v$. We do this probabilistically. Our main tool will be Hoeffding's Inequality [13] applied to the binomial distribution. For a random variable $X$ distributed as $\operatorname{Bin}(n, p)$, Hoeffding's Inequality states that $\operatorname{Pr}[X \leq k] \leq \exp \left(-2(n p-k)^{2} / n\right)$.

From each block in the Steiner system, choose an element uniformly at random as a candidate to make a barbell. For each $i$, let $X_{i}$ be the number of blocks that choose $v_{i}$. Clearly, $X_{i}$ is distributed as $\operatorname{Bin}(r, 1 / 4)$, where $r=(m-1) / 3$, with $E\left[X_{i}\right]=r / 4$. Let $A_{i}$ denote the 
event $X_{i} \leq \frac{r}{4}-\sqrt{r \log r}$. By Hoeffding's Inequality, $\operatorname{Pr}\left[A_{i}\right] \leq \exp \left(-2(\sqrt{r \log r})^{2} / r\right)=1 / r^{2}$. When $m \geq 11$,

$$
\operatorname{Pr}\left[\bigvee_{i=1}^{m} A_{i}\right] \leq \sum_{i=1}^{m} \operatorname{Pr}\left[A_{i}\right] \leq \frac{m}{r^{2}}=\frac{9 m}{(m-1)^{2}}<1
$$

Thus some outcome of the experiment chooses each vertex at least $r / 4-\sqrt{r \log r} \operatorname{times}$.

By combining blocks in pairs, we save at least $\frac{1}{2}(r / 4-\sqrt{r \log r})$ bars for each vertex. As noted earlier, the bars for $v_{n+1}, \ldots, v_{m}$ can then be deleted. Thus

$$
b\left(T_{n}\right) \leq r-\left(\frac{r}{8}-\frac{\sqrt{r \log r}}{2}\right) \leq \frac{7(n+7)}{24}+\frac{1}{2} \sqrt{\frac{n+7}{3} \log \left(\frac{n+7}{3}\right)} \leq \frac{7}{24} n+2 \sqrt{n \log n} .
$$

When $n$ is sufficiently large, the upper bound can be improved further.

(Theorem 1.10(b)). If $n$ is sufficiently large, then $b\left(T_{n}\right)<3 n / 14+42$.

Proof. By Theorem 4.2, a Steiner system $S(2,15, n)$ exists when $n=210 s+1$ or $n=210 s+15$, where $s$ is larger than some constant $n_{0}$. Therefore, for sufficiently large $n$ there is a Steiner system $S(2,15, m)$ with $n \leq m \leq n+195$. Each vertex appears in $(m-1) / 14$ blocks, and in the layout of each one according to Figure 5.2 it gets three bars. Thus

$$
b\left(T_{n}\right) \leq b\left(T_{m}\right) \leq \frac{3}{14}(m-1) \leq \frac{3}{14}(n+194)<\frac{3}{14} n+42 .
$$

As noted earlier, the argument generalizes. If there is a $k$-fold layout of a transitive $\ell$-tournament, then $b\left(T_{n}\right) \leq k n /(\ell-1)+O(1)$. The planarity restriction requires $\ell \leq 6 k+1$, but we do not know the best bound that can be obtained by this method.

\section{Open Questions}

We have developed bounds on visibility number of oriented graphs in terms of decomposition parameters (arboricity) and interval number. We presented upper bounds for planar graphs, outerplanar graphs, bipartite graphs, and tournaments. We conclude by collecting the open problems posed along the way, for convenient reference.

1. Conjecture: If $G$ is an orientation of an undirected graph $H$ then $b(G) \leq 2 b(H)$.

2. Is there a planar digraph $G$ with $b(G)=4$ ?

3. Is there an orientation $G$ of the $d$-dimensional hypercube with $b(G)=\lceil(d+1) / 4\rceil$ ?

4. Over all digraphs $G$, what is the smallest $\alpha$ such that always $b(G) \leq \alpha n+c$ for some constant $c$, where $n=|V(G)|$ ?

5. What is the best $\alpha$ in Question 4 when $G$ is restricted to be a tournament?

6. What is $\lim _{n \rightarrow \inf } b\left(T_{n}\right) / n$, where $T_{n}$ is the transitive $n$-tournament? (It is at least $1 / 6$.)

7. Conjecture: $b\left(T_{11}\right)=3$. Also, is it true that $b\left(T_{16}\right) \geq 4$ ? 


\section{References}

[1] J. Balogh, M. Kochol, A. Pluhar, and X. Yu. Covering planar graphs with forests. J. Combin. Theory, Ser. B, 94:147-158, 2005.

[2] G. Di Battista, P. Eades, R. Tamassia, and I. G. Tollis. Graph Drawing, Algorithms for the Visualization of Graphs. Prentice Hall, Upper Saddle River, NJ, 1999.

[3] G. Di Battista and R. Tamassia. Algorithms for plane representations of acyclic digraphs. Theoretical Computer Science, 61:175-198, 1988.

[4] T. Beth, D. Jungnickel, and H. Lenz. Design Theory. Cambridge Univ. Press, 2nd edition, 1999.

[5] P. Bose, A. M. Dean, J. P. Hutchinson, and T. Shermer. On rectangle visibility graphs. In S. North, editor, Graph Drawing, Proc. of Symp. on Graph Drawing GD '96, Lecture Notes in Computer Science, pages 25-44. Springer, 1997.

[6] W. Cao. Some Problems in Structural Graph Theory. PhD thesis, U. Illinois, 2006.

[7] Y.-W. Chang, J. P. Hutchinson, M. S. Jacobson, J. Lehel, and D. B. West. The visibility number of a graph. SIAM J. Discrete Math., 148(3):462-471, 2004.

[8] M. R. Garey, D. S. Johnson, and H. C. So. An application of graph coloring to printed circuit testing. IEEE Trans. Circuits Syst., 23:591-599, 1976.

[9] J. E. Goodman and J. O'Rourke. Handbook of Discrete and Computational Geometry. CRC Press LLC, Boca Raton, FL, 2nd edition, 2004.

[10] J. R. Griggs and D. B. West. Extremal values of the interval number of a graph. SIAM J. Algebr. Disc. Meth., 1:1-7, 1980.

[11] H. Hanani. The existence and construction of balanced incomplete block designs. Ann. Math. Stat., 32:361-386, 1961.

[12] P. J. Heawood. Map colour theorem. Quart. J. Pure and Applied Math., 24:332-333, 1890.

[13] W. Hoeffding. Probability inequalities for sums of bounded random variables. J. Amer. Stat. Assoc., 58(301):13-30, 1963.

[14] J. P. Hutchinson, T. Shermer, and A. Vince. On representations of some thickness-two graphs. In F. Brandenburg, editor, Graph Drawing, Proc. of Symp. on Graph Drawing GD '95, Lecture Notes in Computer Science, pages 324-332. Springer, 1996. 
[15] B. Jackson and G. Ringel. Solution of Heawood's empire problem in the plane. J. Reine Angew. Math., 347:146-153, 1983.

[16] S.-J. Kim, A. V. Kostochka, D. B. West, H. Wu, and X. Zhu. Decomposition of sparse graphs into forests and a graph with bounded degree. Submitted.

[17] D. G. Kirkpatrick and S. K. Wismath. Determining bar-representability for ordered weighted graphs. Comp. Geom., 6:99-122, 1996.

[18] D. J. Kleitman. Partitioning the edges of a girth 6 planar graph into those of a forest and those of a set of disjoint paths and cycles. Unpublished manuscript, 2006.

[19] H. Kolb, D. B. West, and J. I. Wise. Visibility number of hypercubes. In preparation.

[20] A. V. Kostochka and D. B. West. Every outerplanar graph is the union of two interval graphs. Congr. Numer. (30th SE Conf. Comb. Graph The. Comp.), 139:354-358, 1999.

[21] M. Montassier, P. Ossona de Mendez, A. Raspaud, and X. Zhu. Decomposing a graph into forests. Unpublished manuscript, 2010.

[22] C. St. J. A. Nash-Williams. Decomposition of finite graphs into forests. J. London Math. Soc., 39:12, 1964.

[23] J. O'Rourke. Art Gallery Theorems and Algorithms. Oxford Univ. Press, Oxford, 1987.

[24] C. Payan. Graphes equilibre et arboricité rationnelle. European J. Combinatorics, 7:263-270, 1986.

[25] C. Reid and A. Rosa. Steiner systems $S(2,4, v)$ - a survey. Elec. J. Combin., Dynamic Survey DS18, accessed 11/01/2010.

[26] E. Scheinerman and D. B. West. The interval number of a planar graph: three intervals suffice. J. Combin. Theory, Ser. B, 35:224-239, 1983.

[27] R. Tamassia and I. G. Tollis. A unified approach to visibility representations of planar graphs. Discrete Comput. Geom., 1(4):321-341, 1986.

[28] W. T. Trotter and F. Harary. On double and multiple interval graphs. J. Graph Theory, 3:205-211, 1979.

[29] J. D. Ullman, editor. Computational Aspects of VLSI Design. Computer Science Press, Rockville, MD, 1984.

[30] R. M. Wilson. An existence theory for pairwise balanced designs. III. Proof of the existence conjectures. J. Comb. Th. (A), 18:71-79, 1975. 
[31] S. K. Wismath. Characterizing bar line-of-sight graphs. In Proc. 1st Ann. ACM Symp. Comput. Geom., SCG '85, pages 147-152, New York, 1985. ACM.

[32] S. K. Wismath. Bar-representable visibility graphs and a related network flow problem. PhD thesis, U. British Columbia, 1989. 